PanAfrican
8gomedical
8080Journal

Case report, Volume 5, Issue 5, 2010
Provisional PDF

Published 24 April 2010

\title{
Foreign body causing perforation of the appendix in an African boy
}

\author{
Peter Hulme $e^{1, \&}$ \\ ${ }^{1}$ Trafford General Hospital, Moorside Road, England
}

\section{${ }^{\text {\& } C o r r e s p o n d i n g ~ a u t h o r ~}$}

Trafford General Hospital, Moorside Road, Davyhulme, Manchester, England, M41 5SL. peterhulme@doctors.org.uk

\begin{abstract}
Foreign bodies in the appendix are a rare but well described clinical entity and may cause perforation. Presented here is the case of a 13yr old Ugandan boy who had features of acute appendicitis, was sent for appendicectomy and during the operation was found to have perforation of the appendix due to a seed. The boy was treated with broad spectrum antibiotics and made an uneventful recovery.
\end{abstract}

Key-words: Appendicitis, foreign body, Africa, paediatrics, perforation

\section{Background}

Foreign bodies in the appendix are well recognised but rarely found. The author is not aware of any other reported cases of a foreign body causing perforation of the appendix in a child in Africa. Presented here is the case of a 13yr old Ugandan boy with abdominal pain who was found to have a perforated appendix with a seed sitting in the perforation. 


\section{Patient and case report}

A 13 years old male presented to hospital with intermittent abdominal pains for one month associated with watery diarrhoea and mild fever. He had recently taken a course of albendazole. Examination revealed mild tenderness in the right iliac fossa (RIF) and right upper abdomen. A stool sample was negative for parasites and oocysts. It was thought that he was having acute appendicitis and was admitted to the surgical ward. He was treated with oral metronidazole and ciprofloxacin but after two days his symptoms improved and he was discharged home.

Eighteen days later he re-attended surgical clinic complaining of right sided abdominal pain. Again he was tender in the RIF but there was no guarding or signs of peritonism. He was readmitted to the surgical ward and again put on oral metronidazole and ciprofloxacin. His $\mathrm{Hb}$ was $8.6 \mathrm{~g} / \mathrm{dl}$.

Three days after admission the patient was sent for appendicectomy. On the day of the operation his abdomen was soft with mild RIF tenderness and active bowel sounds. There were no features of peritonism. The appendix was located via a grid iron incision and was found to be laying retrocaecally. It was inflamed with a distal perforation and an oval shaped, $13 \times 8 \mathrm{~mm}$ seed was seen to be partially protruding from the perforation (Figure 1). No pus was seen in the abdomen. Peritoneal lavage was performed with warmed $0.9 \%$ saline. The patient was put on X-Pen $2 \mathrm{mu}$ and gentamicin $160 \mathrm{mg}$ with IV fluids. The patient made an uneventful recovery and was discharged home three days post-op in a stable condition. The patient didn't recognise the seed nor could he recall eating it. The seed was sent to Makerere University and the photograph showed to local people but it was not able to be identified.

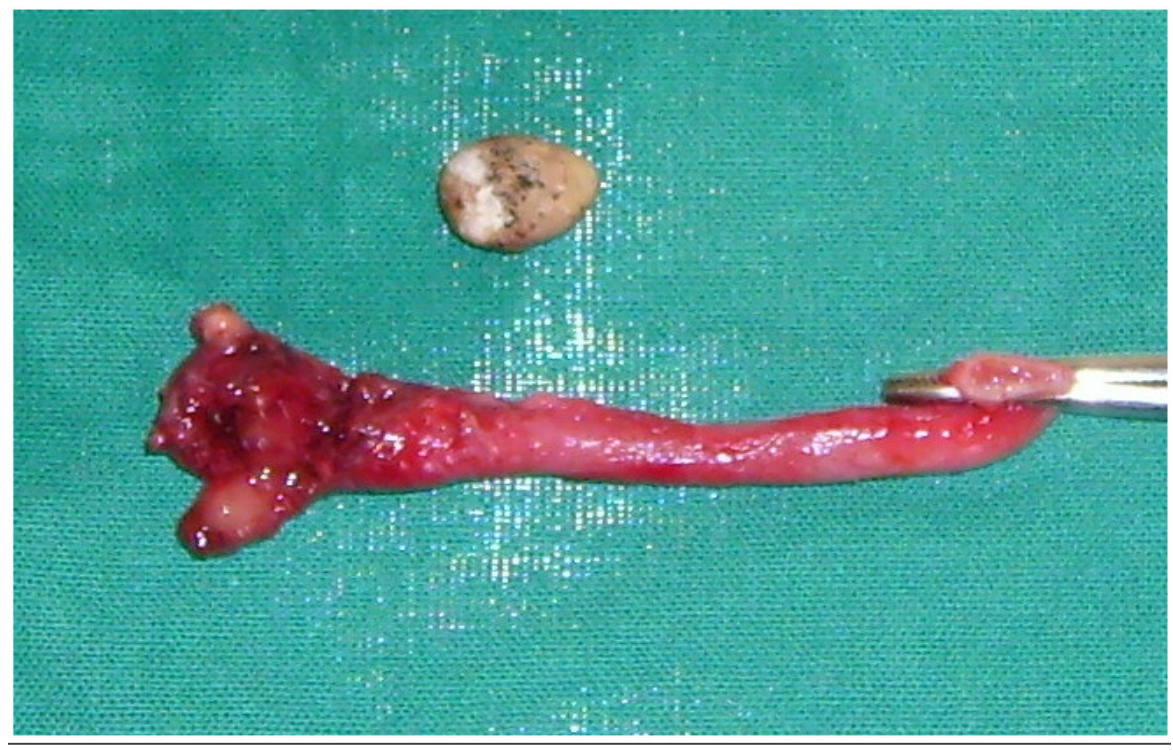

Figure 1

The perforated appendix and seed 


\section{Discussion}

Abdominal pain in children is a common problem seen by doctors all over the world. Pain in the abdomen has a broad differential diagnosis encompassing relatively benign conditions such as UTI or constipation to life threatening emergencies such abdominal perforation [1]. Appendicitis is the most commonly performed emergency abdominal surgery and the leading cause of acute paediatric surgical admission yet determining which patients have appendicitis is difficult [2,3]. Various scoring systems based on clinical signs have been shown to be of use in identifying patients at high risk of appendicitis [4].

Foreign bodies in the appendix are very uncommon with one study showing a frequency of $0.005 \%$ in 13,228 patients [4]. The literature has described many causes of foreign bodies in the appendix such as seeds, needles, tongue studs, parasitic worms, bullets and dental drill pieces $[5,6]$. Long, thin, sharp and pointed items are thought to be most likely to cause perforation after ingestion whilst seeds are thought to present a medium risk of perforation [5]. Appendicitis can even occur after appendicectomy, a phenomenon known as 'stump appendicitis' so surgeons still need to consider a diagnosis of appendicitis in patients who have had their appendix removed [7].

\section{Conclusion}

Acute appendicitis is a surgical emergency and delay in performing appendicectomy increases the risk of perforation. Perforation of the appendix due to a foreign body can occur without classical signs and symptoms of perforation. Surgeons all over the world should be aware of the potential operative findings of a foreign body and be able to deal with them appropriately.

\section{Consent}

The patient and his family gave consent for the article to be written.

\section{Conflict of interest}

The author declared they have no competing interests. 


\section{References}

1. Bundy DG, Byerley JS, Liles EA, Perrin EM, Katznelson J, Rice HE. Does this child have appendicitis?. JAMA. 2007 Jul 25;298(4):438-51. This article on PubMed

2. Marudanayagam R, Williams GT, Rees BI. Review of the pathological results of 2660 appendicectomy specimens. Journal of Gastroenterology. 2006; 41 (8): 745-9. This article on PubMed

3. Pfeil M, Mathur A, Singh S, Morris C, Green G, Kulkarni M. Home next day: early discharge of children following appendicectomy. Journal of Child Health Care. 2007; (3): 208-20. This article on PubMed

4. Attia MW. The Paediatric Appendicitis Score (PAS) was useful in children with acute abdominal pain. Evidence Based Medicine. 2009;14(1):26. This article on PubMed

5. Klingler PJ, Seelig MH, DeVault KR, Wetscher GJ, Floch NR, Branton SA, Hinder RA. Ingested foreign bodies within the appendix: A 100-year review of the literature. Dig Dis. 1998 SepOct;16(5):308-14. This article on PubMed

6. Fischer $C D$, Mukherjee $A$. Appendicitis due to tongue stud ingestion: a case study and review of management plans. South Dakota Journal of Medicine. 2004;57(1):19-22. This article on PubMed

7. Waseem M, Devas G. A child with appendicitis after appendectomy. Journal of Emergency Medicine. 2008;34(1):59-61. This article on PubMed 